Steinzellen sind hier keine sicheren Anhaltspunkte zur Unterscheidung von echten Zimmtrinden. Die Steinzellen und Stärkekörner sind indessen im Allgemeinen im mikroskopischen Bilde weniger zahlreich vorhanden, als es beim echten Zimmt der Fall ist. Das Vorhandensein von Oxalatprismen gestattet diese Rinde in eine bestimmte Gruppe von Zimmtrinden einzureihen. Da einerseits primäre Faserbündel fehlen, andererseits in der sekundären Rinde die Steinzellen nicht vorhanden sind, so findet man viel seltener als beim echten Zimmt Bastfasern, denen sklerosirte Elemente angelagert sind. Auffälliger sind die gröberen mikroskopischen Fragmente der Innenrinde, denen im Vergleich zu echtem Zimmt eine grössere Dünnwandigkeit und geringere Färbung des Parenchyms zukommt, welche nach Behandlung des ursprünglichen Rindenpulvers mit verdünnter Salzsäure und Kalilauge noch mehr zum Ausdrucke kommt. Die grosslumigen Schleimzellen erscheinen in solchen Fragmenten oft in Reihen angeordnet (Fig. 19). Porenzellen, wie schon erwähnt, fehlen dieser Rinde.

Viel schwieriger gestaltet sich die Untersuchung, wenn Gemische dieser Rinde mit echten Zimmtrinden vorliegen, da die aufgezählten Merkmale mit denen von manchen echten Zimmtrinden vielfach zusammenfallen. Diese Merkmale der beschriebenen Rinde sind zu wenig charakteristisch, um in jedem Falle die Beimischung derselben zu echtem Zimmt in Pulver mit Sicherheit feststellen zu können. Dass sie aber dennoch von Werth sind, ergiebt die Thatsache, dass ich aus der Untersuchung des fraglichen Zimmtpulvers die fremdartige Beimischung erkannte. Dieser Umstand nebst noch anderen Gründen veranlassten die hiesige Untersuchungsanstalt, an der Ursprungsstelle eine Nachforschung vornehmen zu lassen, die zum Funde dieser Rinde führte.

\title{
Allgemeiner Gang zur Untersuchung der Kautschukwaaren.
}

$$
\text { Von }
$$

\section{Octave Chéneau in Paris.}

Ausser ganz oder theilweise vulkanisirtem Gummi, dem Vulkanisirungsschwefel, der sich theils frei, theils an organische Substanzen gebunden vorfindet, und Farbstoffen, wie Ocker, Ultramarin und Zinnober, können die Kautschukwaaren Fremdkörper enthalten, die entweder dazu bestimmt sind, ihnen gewisse Eigenschaften für ihre besondere Verwendung zu verleihen, oder um ihren Preis - meist auf Kosten ihrer Güte - herabzusetzen. Solche Körper, denen man zumeist begegnet, sind: Kalk, Kreide, Zinkoxyd, Gyps, Schwerspath, Talk, Kaolin, Bleiglätte, Mennige, Bleiweiss, Kartoffelmehl, aufgearbeiteter und wiederbenutzter Kautschuk, künstliche Ersatzmittel — sogenannte Faktis -, oder trocknende, durch längeres Kochen mit Oxydationsmitteln, oder durch Behandeln mit Chlorschwefel verdickte Oele; kleine Mengen unverseifbare Oele - Mineral- oder Harzöle - begleiten gewisse Sorten Kunstprodukte und die meisten aufgearbeiteten alten Kautschuke, um deren Erweichung und das Zusammenkneten zu erleichtern; seltener findet man geraspelten Kork, Leder, Pflanzenfasern, Asbest, Graphit oder Pech. 
Die Hartkautschuke können mit Asphalt, Harz oder Ozokerit vermischt sein.

Es empfiehlt sich, die Muster verschiedenen physikalischen Proben zu unterwerfen, wie Zug, Druck, Wirkung fetter Oele und besonders der Einwirkung trockener Hitze bei $135^{\circ}$ und des Wassers bei $170^{\circ}$, wie solche von C. A. Lobry de Bruyn und F. H. van Leent ${ }^{1}$ ) angegeben wurden; derartige Vorversuche ergeben oft verwendbare Anhaltspunkte.

Die chemische Untersuchung der Kautschukwaaren gestaltet sich, in Folge der grossen Zahl von Fremdkörpern, die dem Gummi beigemischt sein können, ziemlich verwickelt; die Analyse ergiebt meist nur annähernde Werthe, da sich die verschiedenen Bestandtheile der untersuchten Mischung nur sehr schwer in sicherer Weise und ohne Veränderung trennen lassen. Der Chemiker soll aus den Untersuchungsergebnissen die erforderlichen Schlüsse für die Beurtheilung ziehen und sich nicht mit den nackten Zahlen zufrieden geben, die bei der Untersuchung erhalten wurden.

Ich habe versucht nach folgendem allgemeinen Gange, der wohl in vielen Fällen vereinfacht werden kann, die grösste Zahl der Fremdkörper zusammenzufassen.

\section{Uebersicht.}

I. Feststellung der Einheitlichkeit. Specifisches Gewicht. Asche. Qualitative Analyse der Mineralstoffe.

II. Bestimmung der Löslichkeit in Alkohol. Harzkörper.

III. Bestimmung des Gesammtschwefels und der Mineralstoffe.

IV. Behandlung mit verdünnter Essigsäure unter Zuckerzusatz.

Der Rückstand dient zur $\quad \begin{gathered}\text { Jn Lösung gehen Kalk-und Blei } \\ \text { aus Karbonaten, Mennige etc. }\end{gathered}$

\section{Bestimmung des Schwefels Behandlung mit alkoholischer Natronlauge}

Der Rückstand wird mit Aceton behandelt

$\overbrace{\text { Der Rückstand wird in In Lösung gehen }}$

der Kälte mit Nitrobenzol unverseifbare

behandelt

Oele

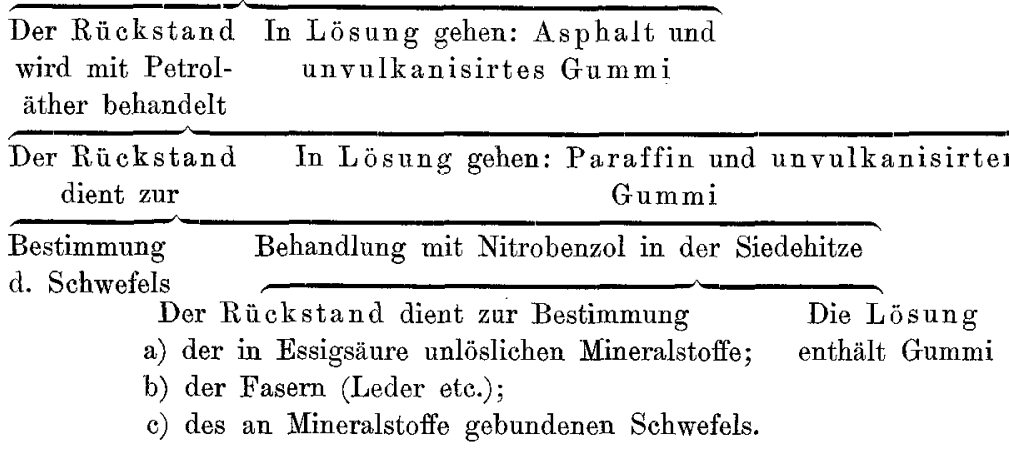

In Lösung gehen: Faktis, freier oder mit Faktis verbundener Schwefel, Harze etc.

V. Bestimmung verschiedener Substanzen. Kohlensäure, Quecksilber etc. 


\section{Feststellung der Einheitlichkeit.}

Man prüft die Einheitlichkeit einer Waare durch Herausgreifen mehrerer Proben, deren specifisches Gewicht und Aschengehalt man bestimmt: die Ergebnisse sollen möglichst übereinstimmen.

Specifisches Gewicht. Bei genügend grossen Proben kann man dasselbe nach dem hydrostatischen Verfahren bestimmen; bei kleinen Bruchstücken benutzt man die Schwimmprobe in mehr oder weniger verdünntem Alkohol, oder man verwendet ein Pyknometer, wobei entweder durch Auskochen mit Wasser, oder durch Stehenlassen im Vakuum die Luftblasen zu entfernen sind. Manche Verwaltungen fordern ein specifisches Gewicht von 0,919-0,956; ohne besonderen Werth auf diese Bestimmung zu legen, kann man annehmen, dass ein erhöhtes specifisches Gewicht einen mehr oder weniger hohen Gehalt an Mineralbestandtheilen verräth. Die Aschenbestimmung giebt für die Beurtheilung der Einheitlichkeit bessere Anhaltspunkte als die Bestimmung des spezifischen Gewichtes, vorausgesetzt, dass keine flüchtigen Aschenbestandtheile, wie Zinnober, zugegen sind.

Asche. Man giebt eine bestimmte Gewichtsmenge Kautschuk in einen weiten bedeckten Porzellantiegel, erhitzt gelinde und entzündet die entweichenden brennbaren Gase; nach dem Verlöschen der Flamme vollendet man die Veraschung unter Luftzutritt bei möglichst niedriger Temperatur und wägt den Rückstand. Bei aschenarmen Kautschuken, oder solchen, die Talk, Kaolin oder Schwerspath enthalten, ergiebt die Aschenbestimmung direkt die Menge der Mineralstoffe; anders ist es beim Vorhandensein flüchtiger, oder durch die Gegenwart reducirender Körper beim Veraschen veränderter Substanzen - Bleiund Quecksilberverbindungen. Im Allgemeinen muss bei erhöhtem specifischem Gewicht und reichlich vorhandener Asche eine vollständige quantitative Analyse der Mineralstoffe ausgeführt werden.

Man verkohlt einfach eine besondere Probe unter Vermeidung der Veraschung, bis kein entzündbares Gas mehr entweicht, und bestimmt im Rückstand qualitativ die Mineralsubstanzen; im unverbrannten ursprünglichem Kautschuk bestimmt man gemeinsam die in verdünnter, mit Zucker versetzter Essigsäure löslichen, ferner die darin unlöslichen Körper und, getrennt, Kohlensäure, Quecksilber und die verschiedenen Elemente.

\section{Bestimmung der Löslichkeit in Alkohol.}

Man erschöpft eine bestimmte Menge Kautschuk mit kaltem oder heissem Alkohol, filtrirt nach dem Erkalten und verdampft das Lösungsmittel. Man entfernt so das Pech, die Harzkörper und einen Theil des Asphaltes. Jede Probe, die ein $2 \%$ überschreitendes alkoholisches Extrakt liefert, kann als mit fremden organischen Stoffen versetzt, oder als geringwerthige Waare angesehen werden. Diese vorläufige Probe wird durch das Aufsuchen verseifbarer Substanzen - Asphalt u. s. w. - ergänzt. 
III. Bestimmung des Gesammtschwefels.

Das rascheste und brauchbarste Verfahren hierzu ist das von R. Henriques ${ }^{1}$ ) empfohlene: Man trägt $1 \mathrm{~g}$ fein zerschnittenen Kautschuk allmählich in $20 \mathrm{ccm}$ heisse reine Salpetersäure ein, verdampft zur Syrupdicke, wenn die Einwirkung beendet ist, fügt dann nochmals $10 \mathrm{ccm}$ Salpetersäure hinzu, verdampft wieder zum Syrup, mischt diesen mit einem feingepulverten Gemenge aus 5 Theilen Natriumkarbonat und 3 Theilen Kaliumnitrat, trocknet die Masse im Wasserbade völlig ein, bringt sie dann in einen Platintiegel unter Ausspülen der Schale mit dem Salzgemisch, das man zum Bedecken der im Platintiegel befindlichen Masse benutzt - im Ganzen etwa $5 \mathrm{~g}$ der Salzmischung - und erbitzt den bedeckten Tiegel allmählich bis zum Schmelzen des Inhaltes; dann lässt man erkalten, nimmt die Schmelze mit Wasser auf und filtrirt. Die Hauptmenge der Schwermetalle bleibt als Oxyd oder Karbonat auf dem Filter, der Gesammtschwefel geht als Sulfat ins Filtrat; vorkommenden Falles auch Kieselsäure und Chrom.

Man kann nach diesem Verfahren gewisse mineralische Elemente zugleich mit.dem Schwefel bestimmen. Man findet auf diese Weise den Gesammtschwefel d. h. den freien sowie den an organische und Mineralsubstanzen gebundenen Schwefel.

Zur Bestimmung des Schwefels in aschenarmen Kautschuken habe ich mich mit Vortheil der Mahler'schen Bombe bedient. In diesem Falle bringt man ungefähr $50 \mathrm{ccm}$ Wasser in die Bombe, beschickt den Platintiegel mit 1-2 g Kautschuk, schraubt den Deckel auf, lässt Sauerstoff unter 5-10 Atmosphären Druck eintreten und bewirkt die Entzündung des Kautschuks durch einen den Kautschuk berührenden, durch den elektrischen Strom glühend gemachten feinen Platindraht; von der stattgehabten Verbrennung überzeugt man sich durch Auflegen der Hand auf die Bombe, die sich dabei erhitzt. Nach einigen Minuten schüttelt man die Bombe, damit das Wasser die entstandene Schwefelsäure völlig aufnimmt, entleert sie, spült sie mit Wasser aus, das man der ersten Flüssigkeit zufügt und fällt die Schwefelsäure als Baryumsulfat. Das Verfahren ist rasch ausfübrbar and ergiebt mit binreichender Genauigkeit den Gehalt an freiem und an organische Stoffe gebundenem Schwefel aschenarmer Kautschuke.

\section{Bestimmung der in verdünnter Essigsäure löslichen Bestand theile.}

Eine bestimmte Gewichtsmenge kleingeschnittenen Kautschuks wird in der Wärme mit verdünnter Essigsäure behandelt, die mit Zucker versetzt ist, um die Mennige zu lösen; Kartoffelmehl, Kalk, Kreide, Bleiweiss, Mennige, Zinkoxyd und die Alkalien gehen in Lösung. Wenn die Probe mit Ultramarin gefärbt war, wird die blaue Farbe zerstört, ein Theil des Ultramarinschwefels

1) Zeitschr. angew. Chem. 1899, 802-840; diese Zeitschrift 1900, 3, 210. 
entweicht in Form von Schwefelverbindungen, ein Theil bleibt in der Flüssigkeit suspendirt und geht beim'Filtriren durchs Filter. Die Menge in Essigsäure löslicher Verbindungen, die hierbei im Kautschuk zurückbleiben, ist nur gering und kann vernachlässigt werden. Man giesst auf ein gewogenes Filter ab, kocht den Rückstand zwei- oder dreimal mit Wasser aus, das man durch das Filter giesst, bringt dann den Rückstand aufs Filter, wäscht ihn mit Wasser, bis die ablaufende Flüssigkeit neutral reagirt, trocknet bei $105^{\circ}$ und wägt. Die essigsaure Lösung kann zur Bestimmung von Kalk, Blei, Magnesia, Zink, Alkalien u. s. w. dienen. Diese Behandlungsweise gestattet die Form zu bestimmen, in der sich die Mineralsubstanzen finden.

1. Bestimmung des Schwefels. Wenn die Behandlung mit Essigsäure ein Entweichen von Schwefelwasserstoff hervorruft, aber nur in diesem Falle, bestimmt man den Schwefel in einem aliquoten Theile des Rückstandes; aus der Differenz vom Gesammtschwefel erhält man den Ultramarinschwefel und den Schwefel in Form von durch verdünnte Essigsäure angreifbaren Verbindungen.

2. Behandlung mit alkoholischer Natronlauge. Eine mit verdünnter und mit Zucker versetzter Essigsäure behandelte und ausgewaschene Probe wird zwei bis drei Stunden lang mit einer Normalnatronlösung in $95 \%$-igem Alkohol am Rückflusskühler gekocht; dann destillirt man das Lösungsmittel $a b$, nimmt mit siedendem Wasser auf und giesst auf ein gewogenes Filter ab; den Rückstand behandelt man zur Vorsicht aufs Neue eine bis zwei Stunden mit alkoholischer Natronlauge, verjagt den Alkohol, wäscht zwei- oder dreimal mit siedendem Wasser, das durch das Filter abgegossen wird, aus, behandelt dann mit Wasser, das mit Essigsäure angesäuert ist, bringt den Rückstand aufs Filter, wäscht ihn mit siedendem Wasser aus, bis das Filtrat nicht mehr sauer reagirt, trocknet bei $105^{\circ}$ und wägt. Der hierbei erhaltene Gewichtsverlust ergiebt die Faktis, freien and an letztere gebundenen Schwefel, die natürlichen Harzbestandtheile des Gummis und die zugesetzten Harzkörper, Pech, Harz u. s. w. Nach R. Henriques ${ }^{1}$ ), der die in vorstehender Weise abgeänderte Behandlung mit alkoholischer Natronlauge empfohlen hat, verlieren reine Kautschuke hierbei 0,9 bis 3,5, im Mittel 2,5\%; nach Terry ${ }^{2}$ and nach Lobry de $B \mathrm{yn}^{3}$ ) ist der Gewichtsverlust gewisser so behandelter Kautschuke sehr schwankend und kann bis $40 \%$ steigen. Die geschätztesten Sorten enthalten wenig Harzsubstanzen und erleiden in Folge dessen einen geringen Gewichtsverlust; man kann, ohne den löslichen Theil im Wesentlichen als Verfälschung zu betrachten, Kautschuke, welche bei dieser Behandlung mehr als 8-10\% an Gewicht verlieren als geringwerthige Sorten ansehen.

a) Behandlung mit Aceton. Eine Probe, an der die vorstehenden Behandlungen ausgeführt sind, wird mit Aceton erschöpft, bis sich das Lösungs-

3) Chem. Ztg. 1894, 18, 411 und 442.

3) Journ. Soc. chem. Ind. 1889, 8, 173-175 und 1892, 11, 970-974.

3) Chem.-Ztg. 1894, 18, 329. 
mittel nicht mehr färbt; dann destillirt man das Aceton ab, nimmt den Rückstand mit Aether auf, filtrirt, dampft ab, trocknet bei $100^{\circ}$ und wägt. Man erhält so die unverseifbaren Oele (Mineral-oder Harzöle) gemischt mit Asphalt, wenn die Probe solchen enthält.

b) Behandlung mit Nitrobenzol in der Kälte. Den mit Aceton erschöpften Rückstand digerirt man nach dem Verfahren von R. Henriques ungefähr eine Stunde lang mit kaltem Nitrobenzol, wobei $30 \mathrm{ccm}$ davon auf $1 \mathrm{~g}$ Substanz anzuwenden sind; man filtrirt unter Auspressen des Gummis mit einem Pistill, wäscht mit Nitrobenzol nach, kocht darauf mit Wasser aus, um die letzten Spuren Nitrobenzol zu entfernen, filtrirt, trocknet und wägt. Unvulkanisirter Gummi verliert dabei $1-2 \%$, vulkanisirter bis $4 \%$, im Mittel $3 \%$; bei Hartgummi ist der Gewichtsverlust ein wenig geringer. Der noch vorhandene Asphalt geht hierbei völlig in Lösung, sowohl wẹnn man es mit natürlichem als auch mit künstlich aus Theer hergestelltem Asphalt zu thun hat.

c) Behandlung mit Petroläther. Der Rückstand der vorigen Operation wird mit Petroläther, niedrigsiedendem. Benzin oder Chloroform erschöpft, um Paraffin und unvulkanisirtes Gummi zu entfernen; letzteres ist schon zum Theil dureh kaltes Nitrobenzol ausgezogen worden, falls es in beträchtlicher Menge zugegen war.

a.) Schwefelbestimmung. In einem aliquoten Theile des vorigen Rückstandes ist der Schwefel zu bestimmen. Aus der Differenz mit der unter IV, 1 angegebenen Schwefelbestimmung erhält man die Menge des freien, sowie des an Faktis und Asphalt gebundenen Schwefels. Nach Helm enthalten die Asphalte $8-11 \%$ an organische Substanz und $0,36-0,4 \%$ an Basen gebundenen Schwefel.

$\beta)$ Behandlung mit Nitrobenzol in der Hitze. Ein aliquoter Theil des nach e) erhaltenen Rückstandes, oder eine mit verdünnter und mit Zucker versetzter Essigsäure behandelte Probe wird ungefähr eine Stunde lang mit einer Mischung von Chloroform und Nitrobenzol am Rückflusskühler gekocht; nach C. $\mathrm{O} . \mathrm{Weber}{ }^{1}$ ) verwendet man $3 \mathrm{cem}$ Chloroform und $50 \mathrm{cem}$ Nitrobenzol auf $3 \mathrm{~g}$ Gummi; nach dem Erkalten setzt man $100 \mathrm{ccm}$ Aether hinzu, filtrirt, wäscht auf dem Filter mit Benzin und Aether aus, trocknet und wägt den unlöslichen Rückstand. Bei Hartkautschuken und bei stark beschwerten Kautschuken kommt es manchmal vor, dass sich das Gummi nur sehr schwer löst und dass die Filtration Schwierigkeiten macht; in diesem Falle behandelt man mehrere Proben getrennt, entfernt den grösseren Theil der Gummilösung nach dem Absetzen durch Abgiessen und verwendet den noch etwas Gummi enthaltenden Rückstand zu den unten beschriebenen Bestimmungen.

$\left.\beta^{1}\right)$ Fremde Substanzen. Der nach $\beta$ ) erhaltene Rückstand enthält dre in verdünnter Essigsäure unlöslichen Mineralstoffe: Talk, Kaolin, Ocker, Schwerspath, Goldschwefel, Zinnober, Bleisulfid, Bleisulfat, die beim Vulkanisiren zugesetzt oder gebildet worden sind, selten Graphit, geraspeltes Leder oder Kork

1) Zeitschr. angew. Chem. 1898, 313-315; diese Zeitschrift 1898, 1, 726. 
Pflanzenfasern u.s.w. In aliquoten Theilen des gleichen Rückstandes nimmt man eine mikroskopische Untersuchung vor, um Fasern aufzusuchen, eine Stickstoffbestimmung, um Leder zu erkennen sowie eine Behandlung mit Salpetersäure durch Abdampfen des Rückstandes zur Trockene und Veraschen, um die Mineralstoffe gemeinsam zu erhalten, wobei zu berücksichtigen ist, dass etwa vorhandenes Blei- und Antimonsulfid in Bleisulfat, bezw. Antimontetroxyd verwandelt und Zinnober verflüchtigt wird.

$\left.\beta^{2}\right)$ Schwefelbestimmung. Eine Schwefelbestimmung in dem nach $\beta$ ) erhaltenen Rückstand ergiebt aus der Differenz mit der Bestimmung nach $\alpha$ ) den Gehalt an Schwefel, der an Kautschuk gebunden war, sowie direkt denjenigen, der an Mineralstoffe gebunden war; man kann gleichzeitig wie nach IV. gewisse Mineralsubstanzen bestimmen.

\section{Verschiedene Bestimmungen.}

Kohlensäure. Die beschwerten Kautschuke enthalten oft Karbonate und es empfiehlt sich die Kohlensäure nach folgendem Verfahren zu bestimmen: In einem mit Kühler versehenen Kolben lässt man verdünnte Salzsäure oder Salpetersäure auf eine bekannte Gewichtsmenge des sehr fein zerschnittenen Kautschuks einwirken; die Einwirkungsprodukte werden durch Ueberleiten über Bimstein, der mit Kupfersulfatlösung und mit Schwefelsäure getränkt ist, von Schwefelwasserstoff und Wasser befreit und dann die Kohlensäure in Natronkalkröhren gebunden, deren Gewichtszunahme die Kohlensäuremenge ergiebt. Bei wenig oder schlecht vulkanisirten Kautschuken kann die Einwirkung der Säuren mangelhaft sein und der Gummi ihrer Wirkung auf die Karbonate hinderlich sein; in diesem Falle erschöpft man die Probe zuerst mit Chloroform und Nitrobenzol in der Hitze und lässt alsdann die Säuren auf den unlöslichen Rückstand einwirken. Wenn der Rückstand pulverig oder gut vom Gummi befreit ist, kann man die Einwirkung in der Kălte vornehmen und die Kohlensäure volumetrisch mit Hülfe eines Kohlensäurebestimmungsapparates - Calcimeter - bestimmen, worin das Wasser durch verdünnte Kupfersulfatlösung ersetzt ist.

Zinnober. Der Zinnober findet sich in den Zahnkautschuken und den feinen Hartkautschuken - Stabilit -, denen er die lebhaft rothe Farbe ertheilt. Wenn die Farbe die Gegenwart von Zinnober vermuthen lässt, bestimmt man das Quecksilber auf trockenem Wege in dem nach IV, $2, b$ ) erhaltenen Rückstande.

Das vorstehend beschriebene Verfahren kann, wie gesagt, in vielen Fällen vereinfacht werden; es kann aber auch durch weitere Untersuchungen ergänzt werden, die man an den verschiedenen isolirten Bestandtheilen ausführt - verseitbare und unverseifbare Körper u.s. w. --, Proben, über die ich mich hier nicht weiter verbreiten kann. 